\title{
Frieden auf Erden
}

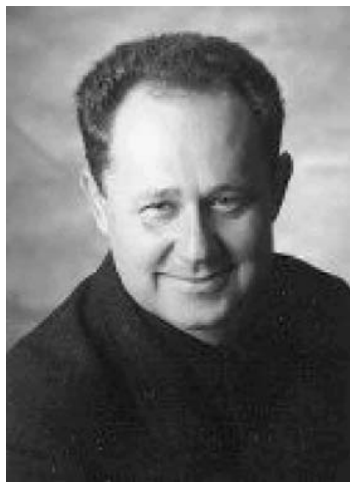

Prof. Dr. Manfred Wildner
Bibliografie

DOI http://dx.doi.org/ 10.1055/s-0030-1269911

Gesundheitswesen 2010; 72: 857-858

(c) Georg Thieme Verlag KG Stuttgart · New York ISSN 0941-3790

Korrespondenzadresse Prof. Dr. M. Wildner Bayerisches Landesamt für Gesundheit und Lebensmittelsicherheit Veterinärstraße 2 85762 Oberschleißheim Manfred.Wildner@lgl.bayern.de
"Et in terra pax hominibus bonae voluntatis" „Und Friede auf Erden allen Menschen guten Willens“, lässt der Evangelist Lukas die Engel über den Hirten auf Bethlehems Feldern singen (Kap. 2, 14). Grund der himmlischen Freude ist ein neugeborenes Kind. Nach heutiger Begrifflichkeit wird dieses (Christ-)Kind durchaus in prekäre Verhältnisse hineingeboren: Die schwangere Mutter - man nimmt ein Alter von 15 Jahren an, also eine Teenage-Schwangerschaft - wurde nach der Überlieferung von einem Mann zur Frau genommen, der nicht der leibliche Vater des Kindes war. Die Geburt findet unter ärmlichsten Umständen statt und mündet zunächst in eine $\mathrm{Mi}$ grantenexistenz (Flucht nach Ägypten). „Meine Augen haben das Heil gesehen“, werden dennoch die Worte des greisen Simeon beim Anblick dieses Kindes im Tempel von Jerusalem überliefert. Ein warmes Willkommen auf dieser Welt, trotz aller äußeren Not.

Menschen werden wohl weltweit und über alle Regionen, Religionen und Regierungsformen hinweg von der Geburt eines Kindes angerührt. Alles menschliche Leben hat auf diese Weise begonnen. Die Menschheit ist auf eine ausreichende Zahl an Neugeborenen angewiesen, welche das Leben in die Zukunft tragen. Der Geburtenüberschuss und das rasante globale Bevölkerungswachstum unserer Tage sind ein Phänomen der neueren Zeit - während der längsten Zeit der menschlichen (Vor-)Geschichte dürfte die Sorge eher dem puren Überleben gegolten haben. In der biblischen Erzählung fand die obige Geburt während einer Volkszählung im Rahmen einer Steuerschätzung statt. Unsere moderne Wissenschaft der Demografie hat weiter reichende Fragestellungen: Wie ist die Lebenserwartung der Menschen? Wie ist die Geburtenziffer gebärfähiger Frauen? Welches sind die zu beobachtenden und die zu erwartenden Wanderungsbewegungen? Ein zentraler Forschungsgegenstand betrifft die Zukunft: wie wird sich die Bevölkerung in 10, 20,30, 50 Jahren entwickelt haben? Im Kontext der sozialen Absicherung kommen weitere Fragen dazu: Wie wird sich der Beitragssatz der gesetzlichen Krankenversicherungen verändern, wie der Steuerzuschuss, wie private Zuzahlungen? Wie entwickelt sich der Lastquotient der Berufstätigen gegenüber den nicht Berufstätigen (Kinder, ältere Menschen)? Sind unsere Renten noch gesichert?

In vielen entwickelten Industrienationen vollzieht sich seit über 100 Jahren ein tiefgreifender demografischer Wandel. Während der Anteil der Über-65-jährigen an der Bevölkerung im Jahr
1900 noch bei $5 \%$ lag, lag er 2010 bereits etwa $20 \%$ und wird voraussichtlich bis 2030 auf etwa $25 \%$ ansteigen. Die Anzahl der hochaltrigen über 85-jährigen darunter wird bis dahin ebenfalls kräftig zunehmen (sog. „Doppelte Alterung“, $[1,2])$. Um einen Eindruck vom - derzeitigen Gesundheitszustand bei den Über-65-jährigen zu geben: in diesem Alter sind im Durchschnitt mehr als 30 ambulante Praxiskontakte jährlich zu verzeichnen. Bluthochdruck, Störungen des Fettstoffwechsels, Rückenschmerzen und Defizite des Sehvermögens sind die wichtigsten Behandlungsanlässe. Hinzu kommen Pflegebedürftigkeit, Altersdemenz und Altersdepression. Im stationären Behandlungsgeschehen stehen HerzKreislauferkrankungen, Krebs, Erkrankungen des Verdauungssystems und Muskel-Skeletterkrankungen - darunter auch die Folgen von Stürzen - an erster Stelle [3,4].

Die dadurch ausgelösten durchschnittlichen jährlichen Behandlungskosten liegen bei den 65-85jährigen doppelt so hoch wie bei den 45-65-jährigen und verdoppeln sich bei den über 85-jährigen noch einmal [5]. Zwei Drittel der über 65jährigen weisen mindestens 2 chronische Krankheiten auf, 35\% der Männer und 40\% der Frauen über 65 Jahren nehmen 9 und mehr Wirkstoffe in Dauertherapie ein [6]. Vertreter der Sterbekostenhypothese weisen darauf hin, dass hohe Krankheitskosten vor allem im letzten Lebensjahr anfallen. Diese Kosten fallen in jüngeren Lebensjahren höher aus als bei Hochaltrigen. Dies wäre in gewisser Hinsicht beruhigend - bei steigender Lebenserwartung wäre damit zu erwarten, dass die Gesundheitsausgaben des jeweils letzten Lebensjahres rückläufig sind. Freilich fallen in den gewonnen Jahren selbst weiter Gesundheitsausgaben an.

Die öffentliche Diskussion dazu scheint angstbesetzt. Wird es in den Systemen der sozialen Sicherung einen Kampf um knappe Ressourcen geben? Wird das Alter mehr und mehr von Verarmung gekennzeichnet sein? Sind die Krankheitskosten noch finanzierbar? Der Sozialstatistiker Gerd Bosbach führt eine ganze Reihe von Argumenten ins Feld, welche helfen, die DemografieDiskussion zu entdramatisieren [7]: Die Wirkung der Arbeitslosigkeit auf die Sozialversicherung übertrifft die der demografischen Entwicklung der kommenden 20 Jahre deutlich. Die derzeitige Geburtenziffer in Deutschland - sie liegt pro Frau während ihres gebärfähigen Alters bei ca. 1,4 Kindern -ist in der Tat recht niedrig. Doch ist der Bezugsrahmen „Deutschland“ in einem sich einigenden Europa für die Zukunft überhaupt zutref- 
fend? Und wie ist die Ressource hochqualifizierter Frauen einzuschätzen, die derzeit aufgrund fehlender Innovationen einer frauenfreundlichen Arbeitsplatz- und Schulgestaltung nicht zum Tragen kommt? Auf der Grundlage der gegenwärtig verfügbaren Daten ist nicht davon auszugehen, dass der demografische Wandel das Gesundheitswesen unbezahlbar macht. Im letzten Jahrhundert stieg die Lebenserwartung um mehr als 30 Jahre - zugegebenermaßen besonders durch eine Sterblichkeitsabnahme im Kindesalter -, bis Mitte des 21. Jahrhunderts wird dem gegenüber nur mit zusätzlich 6 Lebensjahren gerechnet. Auch junge Menschen bedürfen der gesellschaftlichen Unterstützung, z.B. durch Schulen und Universitäten. Der Gesamtquotient der abhängigen Kinder und Älteren gegenüber den Erwerbstätigen stiege, wenn das tatsächliche Renteneintrittsalter statt bei heute 60 Jahren bei den gesetzlich vorgegebenen 65 Jahren läge, nur um $4 \%$. Gegenzurechnen ist eine Steigerung der Produktivität, die konservativ bei über $1 \%$ pro Jahr angenommen werden kann - dies ließe die Leistung jedes Erwerbstätigen in 50 Jahren um über $80 \%$ ansteigen. Auch vollzieht sich das Altwerden heute tendenziell bei besserer Gesundheit als früher [8].

Chronische Krankheiten stehen im Mittelpunkt des Versorgungsgeschehens. Prävention, Rehabilitation und Pflege sowie insbesondere auch eine „sprechende Medizin“ gewinnen dadurch an Bedeutung gegenüber der Hochleistungsmedizin des kurativen Bereichs. Die Chancen der Prävention im Alter, auch zum Erhalt der Fähigkeit zu einer unabhängigen Lebensführung, sind noch nicht ausgeschöpft. Die Medizintechnik kann wertvolle Beiträge für ältere Menschen liefern, z.B. Telemonitoring und Home Health-Ansätze. Integrative Versorgungsformen und Disease-Management-Programme werden zunehmend wichtiger, um eine umfassende Versorgung ohne Brüche in der Versorgungskette sicherzustellen - z.B. an den Schnittstellen Hausarzt/Akut-Krankenhaus/Rehabilitation. Die heimärztliche Versorgung und die Pflegequalität können noch verbessert werden. Angebote der Palliativmedizin können gestärkt und flächendeckend verfügbar gemacht werden, ebenso Angebote der ambulanten und stationären Hospizarbeit. Die Versorgungsforschung zu Behandlungsbedarf und Behandlungsverfahren bei Älteren könnte ausgebaut werden - es gilt, bei allen Maßnahmen nicht nur dem Leben Jahre hinzuzufügen, sondern auch den gewonnen Jahren Leben („Add life to years, not (only) years to life“ [9]).

Dieses Heft greift solche und andere Themen auf: Dialyse und Armutsrisiko, vereinsamtes Sterben in Randgruppen, Mangelernährung in Altenpflegeheimen, Ausgaben der öffentlichen Hand durch den Konsum illegaler Drogen, Versorgungsforschung, integrierte Versorgung, UV-Risikowahrnehmung, Patienteninformation und Kreuzschmerzen, Ansätze der bevölkerungsbezogenen Prävention von Neuralrohrdefekten in Österreich, Gesundheit als höchstes Gut.

Manche Sorge ließe sich wohl auch nehmen, wenn die sich immer weiter öffnende Schere zwischen den Bestverdienenden und den Geringverdienern wieder geschlossen würde. Das Jahresgehalt der Bundeskanzlerin (bei Wikipedia zu erfahren), oder Peter Druckers Faktor 20 als Maß für die Obergrenze der Gehaltsspreizung auch für Angestellte (,, a company's CEO should make no more than 20 times the salary of its lowest-paid worker"
[10]): Vielleicht wären dies Anhaltspunkte für eine gerechte Ressourcenverteilung. In einer Gesellschaft, die den bunt bedruckten Banknoten erst ihren (Tausch-)Wert gibt: Durch deren allgemeine Akzeptanz in Treu und Glauben als Zahlungsmittel für vielfältige Dienstleistungen, von $A$ wie Altenpflege bis $Z$ wie Zahnersatz. Als Tauschmittel für Arbeit, die letztlich von Menschen für Menschen erbracht werden muss.

Vorsicht also vor Panikmache: Wir brauchen vor nichts Angst zu haben außer vor der Angst. Und ein vorsichtiges „cui bono“ - wer zieht womöglich Nutzen aus dieser Art von Demografiedebatte? Hätte der Aufbau eines Kapitalstocks wirklich Vorteile gegenüber unserem bisherigen Umlageverfahren? Kann Geld wirklich „arbeiten“, oder sind es nicht immer Menschen, die dies tun? Menschen, deren kostbare Arbeitskraft über Marktmechanismen von Angebot und Nachfrage mit Preisen verknüpft wird, was einen „Kapitalstock“ sehr fragil machen kann? Es sind die Finanzdienstleister privater Altersvorsorge und mit ihnen die Aktien- und Kapitalmärkte, welche kurz- und mittelfristig Gewinne durch einen frischen Kapitalzustrom erfahren. Vielleicht auch mancher Arbeitgeber, der sich so von einer paritätisch mitfinanzierten Rente abkoppeln möchte. Vielleicht brauchen wir einen neuen Intergenerationenvertrag, der weit über die Regelungen von Sozialversicherung, Rentenalter und Krankheitskosten hinausgeht. Auch vor diesem Hintergrund sei ein heilsames „Fürchtet Euch nicht!“ gesprochen - solange und damit es Menschen guten Willens gibt, welche zur Solidargemeinschaft beitragen, sich für Vollbeschäftigung und die Integration von Migranten, gerechte Löhne und eine bessere Vereinbarkeit der beruflichen und privaten Rollen (nicht nur) der Frauen einsetzen und, last but not least, Investitionen in Kinder als Investition in die Zukunft begreifen. Dann kann der (soziale) Frieden auf Erden ankommen und - hoffentlich - bleiben.

\section{Literatur}

1 Scholz R, Idanov D. Weniger Hochbetagte als gedacht. Demografische Forschung aus erster Hand 5 (1): 4. Max Planck Institut für demografische Forschung, Rostock. 2008

2 Statistische Ämter des Bundes und der Länder. Demografischer Wandel in Deutschland. Heft 2. Auswirkungen auf Krankenhausbehandlungen und Pflegebedürftige im Bund und in den Ländern. Statistisches Bundesamt Wiesbaden. 2008

3 Böhm K, Tesch-Römer C, Ziese T, Hrsg. Gesundheit und Krankheit im Alter. RKI, Berlin; 2009

4 Bayerisches Landesamt für Gesundheit und Lebensmittelsicherheit. Gesundheit im Alter. Gesundheitsreport Bayern 2/2009. http://www.lgl. bayern.de/publikationen/index.htm\#gesundheitsberichterstattung 2009

5 Statistisches Bundesamt. Krankheitskostenrechnung. www.gbe-bund.de

6 SVR. Sachverständigenrat zur Begutachtung der Entwicklung im Gesundheitswesen: Koordination und Integration - Gesundheitsversorgung in einer Gesellschaft des längeren Lebens. Sondergutachten 2009. Kurzfassung. 2009

7 Bosbach G. „Die demografische Entwicklung“ - Mythos und Wirklichkeit. In: Zukunft des Alters: Herausforderungen und Chancen der demografischen Entwicklung. Sozialverband VdK Bayern e.V., München 2008; 14-24

8 Vaupel K. Biodemography of human ageing. Nature 2010; 464: $536-542$

9 World Health Organization. Add life to years. WHO, Copenhagen 1982. URL: http://whqlibdoc.who.int/euro/pre-wholis/ICP_INF_002.pdf Zugriff am 09.10.2010

10 Drucker P. Managing the Non-Profit Organization. Oxford; 1990 